\title{
Variación de la densidad relativa de la madera de Pinus greggii Engelm. del norte de México
}

\author{
Miguel López Locia ${ }^{1}$ \\ Salvador Valencia Manzo ${ }^{1}$
}

RESUMEN

Con el objetivo de conocer la variación de la densidad relativa de la madera (peso anhidro/volumen verde) entre y dentro de poblaciones de Pinus greggii Engelm. del Norte de México, se obtuvieron muestras de madera de árboles de 12 poblaciones de la distribución natural de la especie, en cada una de las cuales se hizo un muestreo de 5 a 11 árboles, de los que se extrajo una viruta de madera a $1.30 \mathrm{~m}$ de altura. Cada muestra se seccionó en tres partes que correspondieron de manera aproximada a madera juvenil, de transición y madura. Los resultados mostraron diferencias estadísticas significativas en la densidad relativa de la madera, la cual presentó un valor de $0.47 \mathrm{~g} / \mathrm{cm}^{3}$, por lo que se puede considerar una madera moderadamente pesada. De la variación total observada en la densidad de la madera, el $13 \%$ es atribuible a diferencias entre poblaciones y el $24 \%$ que se puede atribuir a diferencias entre árboles dentro de poblaciones. También se encontró que la densidad de la madera se correlaciona en forma positiva con altura total del árbol $(r=0.36)$, con altura del fuste limpio $(r=$ 0.23 y con la edad $(r=0.30)$. Los resultados muestran la posibilidad de realizar una selección de la densidad de la madera tanto en poblaciones como en individuos, para programas de mejoramiento genético.

PALABRAS CLAVE:

Pinus greggii, variación, densidad de la madera, Norte de México.

\begin{abstract}
The objective of this study was to know the wood density variability between and within populations of Pinus greggii Engelm. from northern Mexico. Wood samples were obtained at the diameter breast height $(\mathrm{DBH} ; 1.30 \mathrm{~m})$ of five to eleven trees from twelve populations. Each sample was sectioned into juvenile, transition, and mature wood. Specific gravity had a value of 0.47 that was considered as moderately heavy wood. Wood density differed between populations and between trees within populations. Out of total wood density variability, thirteen percent was attributed to differences between populations and twenty four percent was due to differences between trees within populations. It was found that wood density was positively correlated $(r=0.36)$ with total tree height, with the height at the base of the crown $(r=0.23)$, and with tree age $(r=0.30)$. These results indicated that wood density can be used for selection of populations and individuals in a tree genetic improvement program.
\end{abstract}

KEY WORDS:

Pinus greggii, variation, wood density, Northern Mexico. 


\section{INTRODUCCIÓN}

Pinus greggii Engelm. es una especie forestal nativa de México, que se distribuye en pequeños rodales a lo largo de la Sierra Madre Oriental (Martínez, 1948). En años recientes se han realizado diversos estudios sobre su distribución (Perry, 1991), taxonomía (Donahue y López, 1999), variación (Donahue y López, 1995), propagación (Becerra y Plancarte, 1993; Cetina et al., 1999) y fisiología y genética (Vargas y Muñoz, 1988; Ramírez, 1993), entre otros, dado su alto potencial para ser utilizada en plantaciones comerciales (Kietzka et al., 1996), así como por su capacidad para adaptarse en terrenos degradados (Malan, 1994).

Por otra parte, se considera que la variación natural es la materia prima del mejoramiento genético forestal; sin ésta no sería posible producir genotipos con crecimiento rápido, resistentes a plagas y enfermedades $y$ bien adaptados a condiciones ambientales adversas (Nienstaedt, 1990). En México, y para el caso particular de $P$. greggii, se han realizado diversos estudios sobre variación en: conos, semillas (Plancarte, 1990), acículas (Beristáin, 1992) y sobre ensayos de procedencias (Ornelas, 1997). En cuanto a pruebas genéticas, éstas han sido principalmente en características de crecimiento (Hernández, 1995; Serrato, 2000). A su vez, la selección de materiales genéticos de especies y procedencias han proporcionado las mayores ganancias y más fáciles de obtener para plantaciones forerstales (Burley y Wood, 1979; Zobel et al., 1987).

La densidad relativa de la madera es una de las características genéticas y físicas más estudiadas, por la aparente sencillez de su determinación (Kollmann, 1959); asimismo, es factor de importancia económica en la industria forestal, ya que tiene un efecto importante sobre el rendimiento y calidad del producto final (madera aserrada, calidad de la pulpa para papel). Además, es una característica con amplia variación y fuerte heredabilidad (Zobel y van Buijtenen, 1989). Sin embargo, en Pinus greggii poco se conoce sobre la variabilidad y control genético de la densidad de la madera, por lo que su estudio pudiera ser de utilidad para la selección y mejoramiento genéticos.

\section{OBJETIVO}

El objetivo del presente trabajo es determinar el patrón y la magnitud de la variación de la densidad de la madera entre árboles y entre poblaciones de Pinus greggii Engelm.

\section{MATERIALES Y MÉTODOS}

Se utilizó material de 12 poblaciones en los estados de Coahuila y Nuevo León, que se encuentran entre los 24ํ16'22"y los 2528'53" de latitud Norte y entre los 995'262 y los 10056'28" de longitud Oeste; poblaciones que en su mayoría tienen precipitación promedio anual entre 500 y $700 \mathrm{~mm}$, con un clima templado (Tabla 1).

De cada población se seleccionaron 11 árboles, excepto en la del Cerro El Potosí, de la que debido al tamaño pequeño de la población se seleccionaron 5 árboles únicamente. En todos los casos se cuidó que la distancia entre ellos fuera mayor a $50 \mathrm{~m}$. A cada árbol se le midió diámetro de copa, diámetro normal (a 1.3 $\mathrm{m}$ de altura), altura total, altura de fuste limpio y grosor de corteza, además de registrar información general del área.

Posteriormente, de cada árbol se extrajo un cilindro de madera a la altura de $1.30 \mathrm{~m}$, utilizando el taladro de Pressler. Cada muestra se identificó con un número progresivo y se guardó en un popote de plástico para su protección, hasta el traslado al laboratorio. 
Tabla 1. Descripción climática y topográfica de 12 poblaciones de Pinus greggii del Norte de México

\begin{tabular}{|c|c|c|c|c|c|c|c|}
\hline \multirow[t]{2}{*}{ POBLACIÓN } & \multirow[t]{2}{*}{ ESTADO } & LATITUD & LONGITUD & ALTITUD & $\begin{array}{l}\text { TEMP. } \\
\text { MEDIA } \\
\text { ANUAL }\end{array}$ & $\begin{array}{l}\text { PRECIP. } \\
\text { MEDIA } \\
\text { ANUAL }\end{array}$ & $\begin{array}{l}\text { FÓRMULA } \\
\text { CLIMÁTICA }\end{array}$ \\
\hline & & Norte & Oeste & msnm & ${ }^{\circ} \mathrm{C}$ & $\mathrm{mm}$ & \\
\hline $\begin{array}{l}\text { Cañón de los } \\
\text { Caballos }\end{array}$ & Coah. & $25^{\circ} 14^{\prime} 27^{\prime \prime}$ & $100^{\circ} 53^{\prime} 05^{\prime \prime}$ & 2380 & $12-14$ & $500-600$ & $C(E) x^{\prime}$ \\
\hline $\begin{array}{l}\text { Ejido } \\
\text { Cuauhtémoc }\end{array}$ & Coah. & $25^{\circ} 6^{\prime} 18^{\prime \prime}$ & $100^{\circ} 56^{\prime} 28^{\prime \prime}$ & 2470 & $12-14$ & $500-600$ & $C(E) x^{\prime}$ \\
\hline Jame & Coah. & $25^{\circ} 1^{\prime} 36^{\prime \prime}$ & $100^{\circ} 36^{\prime} 41^{\prime \prime}$ & 2450 & $12-4$ & $600-700$ & $C(E) x^{\prime}$ \\
\hline Lirios & Coah. & $25^{\circ} 3^{\prime} 14^{\prime \prime}$ & $100^{\circ} 33^{\prime} 12^{\prime \prime}$ & 2470 & $12-14$ & $500-600$ & $C(E) x^{\prime}$ \\
\hline Puerto Conejo & N.L. & $2528^{\prime} 53^{\prime \prime}$ & $100^{\circ} 34^{\prime} 25^{\prime \prime}$ & 2460 & $10-12$ & $600-700$ & $C\left(w_{1}\right)$ \\
\hline Tejocote & N.L. & $25 \div 6 ’ 20^{\prime \prime}$ & $100^{\circ} 29^{\prime} 19^{\prime \prime}$ & 2450 & $12-14$ & $500-600$ & $C\left(w_{1}\right)$ \\
\hline Santa Anita & Coah. & $25^{\circ} 64^{\prime \prime}$ & $100^{\circ} 34^{\prime} 57^{\prime \prime}$ & 2500 & $12-14$ & $500-600$ & $C(E) x^{\prime}$ \\
\hline Las Placetas, & N.L. & $24^{\circ} 5^{\prime} 37^{\prime \prime}$ & $99^{\circ} 55^{\prime} 26^{\prime \prime}$ & 2320 & $10-12$ & $600-700$ & $C(E)\left(w_{1}\right) x^{\prime}$ \\
\hline Cerro Potosí & N.L. & $24^{\circ} 3^{\prime} 25^{\prime \prime}$ & $100^{\circ} 1^{\prime} 59^{\prime \prime}$ & 2320 & $10-12$ & $600-700$ & $C x^{\prime}$ \\
\hline La Tapona & N.L. & $24^{\circ} 2^{\prime} 35^{\prime \prime}$ & $100^{\circ} 11^{\prime} 59^{\prime \prime}$ & 2100 & $18-20$ & $300-400$ & $\mathrm{BS}_{0} \mathrm{hw}$ \\
\hline Ascensión & N.L. & $24^{\circ} 6^{\prime} 22^{\prime \prime}$ & $99^{\circ} 57^{\prime} 39^{\prime \prime}$ & 2350 & $12-14$ & $500-600$ & $C x^{\prime}$ \\
\hline Sierra Hermosa & Coah. & $25^{\circ} 8^{\prime} 49^{\prime \prime}$ & $100^{\circ} 56^{\prime} 03^{\prime \prime}$ & 2450 & $12-14$ & $500-600$ & $C x^{\prime}$ \\
\hline
\end{tabular}

Fuente: INEGI, 1975; INEGI, 1980; INEGI, 1992a; INEGI, 1992b; INEGI, 1993; SSP., 1981.

En el laboratorio, cada porción de madera fue seccionada en tres partes: la primera del centro a los $6 \mathrm{~cm}$, la segunda de los 6 a los $9 \mathrm{~cm}$ y la tercera el resto (después de los $9 \mathrm{~cm}$ ), las cuales se utilizaron como repeticiones dentro del árbol, de manera aproximada correspondían a madera juvenil, de transición y madura, respectivamente.

A cada parte de las secciones obtenidas se le determinó la densidad relativa (peso anhidro/volumen verde) de la madera, utilizando el método de máximo contenido de humedad (Smith, 1954) y el método empírico (Valencia y Vargas, 1997).

Para estudiar el efecto de la población y de árboles dentro de la población, se consideró un diseño completamente al azar, con efecto anidado o jerárquico, que corresponde al modelo estadístico que se muestra enseguida:

$$
\begin{aligned}
& Y_{i j}=\mu+P_{i}+A_{i(j)}+\varepsilon_{(j k)} \\
& i \quad=1,2,3, \ldots, 12 \text { (poblaciones) } \\
& \text { j } \quad=1,2,3, \ldots, 11 \text { (árboles dentro de } \\
& \text { población) } \\
& k \quad=1,2, \ldots, 3 \text { (secciones: cercana a la mé- } \\
& \text { dula, parte media y parte exterma) } \\
& Y_{i j} \quad=\text { valor de la observación } \\
& \mu \quad=\text { media general } \\
& P_{i} \quad=\text { efecto de la résima población } \\
& A_{i(j)} \quad=\text { efecto del } j \text {-ésimo árbol dentro } \\
& \text { de la résima población (error } \\
& \text { experimental) }
\end{aligned}
$$


De acuerdo con el modelo descrito, se realizaron análisis de varianza y de componentes de varianza para la variable densidad de la madera. Los componentes de los cuadrados medios esperados en el análisis de varianza se muestran en la Tabla 2.

Además se realizaron análisis de correlación de Pearson entre las variables dasométricas del arbolado y de la densidad de la madera.

\section{RESULTADOS Y DISCUSIÓN}

El valor promedio, de todos los individuos, de la densidad relativa (peso anhidro/volumen verde) de la madera fue de $0.47 \mathrm{~g} / \mathrm{cm}^{3}$, con valores mínimo y máximo de 0.40 y $0.58 \mathrm{~g} / \mathrm{cm}^{3}$ y un coeficiente de variación de $7.53 \%$.

El valor promedio estimado de la densidad de la madera, parece ser ligeramente superior al reportado por Valencia et al. (1993), para una plantación joven de Pinus greggii establecida en el estado de México, donde se presentaron valores de 0.40 a $0.47 \mathrm{~g} / \mathrm{cm}^{3}$ y casi igual al reportado por Poynton (1977), cuando $P$. greggi se cultiva como exótica en Sudáfrica.

Es probable que la plantación del estado de México sea de alguna procedencia del centro del país, y de ser así, se sabe que los árboles de dichas procedencias tienen una tasa de crecimiento mayor que las del Norte del país (López-Upton y Donahue, 1995). A su vez, en algunos trabajos sobre tasa de crecimiento y densidad de la madera, se ha encontrado que existe una relación inversa, como dan a conocer Valencia et al. (1996) para Pinus patula, lo cual no siempre es así, ya que en algunos casos no existe relación alguna y en otros, incluso, existe una correlación positiva (Zobel y van Buijtenen, 1989).
El valor promedio estimado de la densidad de la madera para este estudio $\left(0.47 \mathrm{~g} / \mathrm{cm}^{3}\right)$, permitiría clasificarla como una madera "moderadamente pesada", de acuerdo con la clasificación de Markward y Heckt (Echenique y Díaz, 1972). Según una propuesta de Ordoñez et al. (1989) para usos de la madera por sus propiedades mecánicas, pero atendiendo exclusivamen-te a la densidad, los usos recomendados serían como combustible, madera de aserrío, postes, pilotes para minas, mangos de herramientas y artículos deportivos. Sin embargo, también se sabe que es ampliamente utilizada en Sudáfrica como pulpa para papel (Kietzka et al., 1996) y para aserrío (Poynton, 1977).

El coeficiente de variación estimado para la densidad de la madera $(7.53 \%)$ es un valor pequeño al compararse con coeficientes de variación de variables dasométricas, como altura, diámetro y volumen en Pinus patula (Valencia et al., 1996), de variables anatómicas y morfológicas de acículas y conos, como el encontrado para Pseudotsuga (Santos, 1998), incluso de variables de traqueidas, como el largo de traqueidas en Pinus rudis (Rodríguez, 1998) y grosor de la pared celular, también en $P$. rudis (Ibarra, 1999). Sin embargo, al compararse con otros estudios de variación en la densidad de la madera, se observa que es cercano a los valores reportados por otros autores. Por ejemplo, al estudiar la variación entre y dentro de árboles, Trujillo (1999) señala un coeficiente de variación de $6.9 \%$ para Pinus teocote y López (1997) indica uno de $11.5 \%$ para Pinus rudis; mientras que al estudiar la variación entre poblaciones y entre árboles de Pseudostuga, Zúñiga y Valencia (1999) señalan un coeficiente de variación de $9.1 \%$.

Debe tenerse presente que características como densidad de la madera, largo de traqueidas, entre otras, son variables de alta heredabilidad y de alto valor económico (Zobel y van 
Buijtenen, 1989), motivo por el cual, aún cuando su variación sea pequeña, son útiles para programas de mejoramiento genético.

El análisis de varianza mostró diferencias estadísticamente significativas $(P \leq 0.0001)$ entre poblaciones y entre árboles dentro de poblaciones, para la densidad de la madera (Tabla 3), lo cual muestra que existe efecto de la localidad y dentro de ella, como ya se ha señalado en otros trabajos, para Pinus chiapensis (Yáñez y Caballero, 1991) y para Pseudotsuga (Zúñiga y Valencia, 1999), entre otros.

Tabla 2. Componentes de los cuadrados medios esperados en el análisis de varianza de la densidad de la madera a nivel de población y entre árboles dentro de población de

\section{Pinus greggii}

\begin{tabular}{|c|c|c|c|c|}
\hline \multicolumn{2}{|c|}{$\begin{array}{l}\text { FUENTE DE } \\
\text { VARIACIÓN }\end{array}$} & $\begin{array}{l}\text { GRADOS DE } \\
\text { LIBERTAD }\end{array}$ & $\begin{array}{l}\text { CUADRADOS } \\
\text { MEDIOS }\end{array}$ & $\begin{array}{l}\text { COMPONENTES DE LOS CUADRADOS } \\
\text { MEDIOS ESPERADOS }\end{array}$ \\
\hline \multicolumn{2}{|c|}{ Población } & $\mathrm{P}-1$ & CMP & $\sigma^{2} e+k_{1} \sigma^{2} P o b(P o b)+k_{2} \sigma^{2} P o b$ \\
\hline \multicolumn{2}{|c|}{ Arb (Pob) } & $\sum(n i-1)$ & $\mathrm{CM} \mathrm{A}(\mathrm{P})$ & $\sigma^{2} e+k_{3} \sigma^{2} A r b(P o b)$ \\
\hline \multicolumn{2}{|l|}{ Error } & $\sum \Sigma \sum \mathrm{pn}(\mathrm{m}-1)$ & $\mathrm{CMe}$ & $\sigma^{2} e$ \\
\hline $\begin{array}{l}\text { Cme } \\
\text { P } \\
\text { ni }\end{array}$ & $\begin{array}{l}=\text { Cuad } \\
=\text { Cuad } \\
\text { dentr } \\
=\text { Cuad } \\
=\text { No.d } \\
=\text { No.d }\end{array}$ & $\begin{array}{l}\text { ados medios de población } \\
\text { ados medios de árboles } \\
\text { de población } \\
\text { ados medios del error } \\
\text { poblaciones } \\
\text { árboles por población }\end{array}$ & $\begin{array}{ll}k_{1} & = \\
k_{2} & = \\
k_{3} & \text { á } \\
\sigma^{2} \operatorname{Arb}(\mathrm{Pob}) & = \\
\sigma^{2} \mathrm{Pob} & = \\
\sigma^{2} \mathrm{e} & =\end{array}$ & $\begin{array}{l}\text { = media armónica del número de muestras por población } \\
\text { = media armónica del número de muestras y número de } \\
\text { árboles entre población } \\
\text { = media armónica del número de muestras por población } \\
\text { = Varianza del árbol dentro de población } \\
\text { = Varianza de población } \\
\text { = Varianza del error. }\end{array}$ \\
\hline
\end{tabular}

Tabla 3. Cuadrados medios esperados y componentes de varianza de la densidad de la madera de Pinus greggii, a nivel de población y entre árboles dentro de población

\begin{tabular}{|c|c|c|c|c|c|c|c|}
\hline \multicolumn{2}{|l|}{ F.V. } & G.L. & C.M. & Fc & $\operatorname{Pr}>\mathrm{F}$ & $\begin{array}{c}\text { COMPONENTES DE } \\
\text { VARIANZA }\end{array}$ & $\begin{array}{c}\text { COMPONENTE } \\
\text { DE VARIANZA } \\
(\%)\end{array}$ \\
\hline \multicolumn{2}{|c|}{ Población } & 11 & 0.011 & 8.50 & $0.0001^{* *} \quad \begin{array}{l}\sigma \\
3\end{array}$ & $\begin{array}{l}\sigma^{2} e+2.984 \sigma^{2} \text { arb }(p o b)+ \\
31.179 \sigma^{2} \text { pob }\end{array}$ & 13 \\
\hline \multicolumn{2}{|c|}{ Arb(Pob) } & 114 & 0.002 & 2.14 & $0.0001^{* *} \sigma$ & $\sigma^{2} e+2.9754 \sigma^{2} \operatorname{arb}(p o b)$ & 24 \\
\hline \multicolumn{2}{|l|}{ Error } & 249 & 0.001 & & & $\sigma^{2} e$ & 63 \\
\hline $\begin{array}{l}\text { F.V. } \\
\text { G.L. }= \\
\text { C.M. }= \\
\text { FC } \\
\text { Pr }>\text { F }=\end{array}$ & \multicolumn{4}{|c|}{$\begin{array}{l}\text { = Fuentes de variación } \\
=\text { Grados de libertad } \\
=\text { Cuadrados medios } \\
=\text { Valor calculado de "F" } \\
=\text { Probabilidad de error tipo I }(\alpha)\end{array}$} & $\begin{array}{l}\text { Arb(Pob) } \\
\sigma^{2} \mathrm{e} \\
\sigma^{2} \text { pob } \\
\sigma^{2} \text { arb (pob) }\end{array}$ & $\begin{array}{l}\text { = Arbol dentro de población } \\
\text { = Varianza del error } \\
\text { = Varianza de población } \\
\text { = Varianza de árbol dentro d } \\
\text { = Significativo al nivel de prob } \\
(P<0.01) \text {. }\end{array}$ & $\begin{array}{l}\text { ación } \\
\text { ad de } 0.01\end{array}$ \\
\hline
\end{tabular}


A su vez, el análisis de componentes de varianza muestra que, de la variación total de la densidad de la madera, el $13 \%$ es atribuible al efecto de diferencias entre poblaciones, mientras que el $24 \%$ se atribuye al efecto de diferencias entre árboles dentro de poblaciones y el $63 \%$ restante se atribuye al error o una fuente no bien identificada (Tabla 3).

Estos resultados son parecidos al encontrado en Pinus chiapensis (Yáñez y Caballero, 1991) y en Pseudotsuga (Zúñiga y Valencia, 1999), donde el efecto de árboles fue mayor que el de localidades. Lo anterior resulta de interés si se considera que se trata de una característica de valor económico y, de comprobarse una alta heredabilidad, podría ser utilizada en programas de cruzamiento y selección. Para diversas características, se ha encontrado que las diferencias atribuibles al efecto de localidades, por lo general se asocian con la adaptabilidad de las especies, mientras que las atribuibles a árboles están más asociadas con la heredabilidad a nivel individual (Zobel y Talbert, 1988). En este caso, las poblaciones del Tejocote, N.L. y Santa Anita, Coah., presentaron mayor densidad relativa de la madera, que las poblaciones de Las Placetas, Cerro El Potosí, Puerto Conejo y La Tapona, N.L. (Tabla 4), sin que ello signifique que este efecto es heredable.

Pinus greggii es una especie con alto potencial para ser utilizada en plantaciones en sitios marginales o considerados de calidad pobre, dado que se ha encontrado que es tolerante a determinados niveles de sequía (Vargas y Muñoz, 1988) y existen diferencias en sobrevivencia y crecimiento entre procedencias (Capó et al., 1993; Valencia et al., 1993), por lo que los genotipos a buscar o crear mediante la cruza y selección, deberían ser tolerantes a sequía, de rápido crecimiento y con características de la madera de mayor valor económico.

Tabla 4. Prueba de separación de medias para la densidad relativa (peso anhidro/volumen verde) de la madera de poblaciones de Pinus greggii del Norte de México

\begin{tabular}{lccc}
\hline POBLACIÓN & N & $\begin{array}{c}\text { DENSIDAD* } \\
\text { MEDIA } \\
\left(\mathrm{g} / \mathrm{cm}^{3}\right)\end{array}$ & $\begin{array}{c}\text { AGRUPACIÓN } \\
\text { DE TUKEY } \\
(\alpha=0.05)\end{array}$ \\
\hline Tejocote, N.L. & 11 & 0.5094 & $\mathrm{~A}$ \\
\hline Santa Anita, Coah. & 11 & 0.5084 & $\mathrm{~A}$ \\
\hline Ascensión, N.L. & 11 & 0.4825 & $\mathrm{~A} \mathrm{~B}$ \\
\hline Cañón de Caballos, Coah. & 11 & 0.4805 & $\mathrm{~A} \mathrm{~B}$ \\
\hline Jamé, Coah. & 11 & 0.4766 & $\mathrm{~A} \mathrm{~B}$ \\
\hline Sierra Hermosa, N.L. & 11 & 0.4722 & $\mathrm{~A} \mathrm{~B}$ \\
\hline Ejido Cuauhtémoc, Coah. & 11 & 0.4711 & $\mathrm{~A} \mathrm{~B}$ \\
\hline Lirios, Coah. & 11 & 0.4636 & $\mathrm{~A} \mathrm{~B}$ \\
\hline Las Placetas, N.L. & 11 & 0.4604 & $\mathrm{~B}$ \\
\hline Cerro El Potosí, N.L. & 5 & 0.4593 & $\mathrm{~B}$ \\
\hline Puerto Conejo, N.L. & 11 & 0.4589 & $\mathrm{~B}$ \\
\hline La Tapona, N.L. & 11 & 0.4436 & $\mathrm{~B}$ \\
\hline "peso anhidrolvolumen verde & & &
\end{tabular}

*peso anhidro/volumen verde 
La densidad de la madera presentó una correlación positiva significativa $(P \leq$ $0.05)$ con la altura total del árbol $(r=0.36)$, con la altura del fuste limpio $(r=0.23)$ y la edad $(r=0.30)$, valores que pueden considerarse como ligeros a moderados. Sin embargo, no se encontró correlación de la densidad de la madera con el diámetro normal.

Se observa que la densidad de la madera se relaciona principalmente con la altura total del arbolado, y ésta a su vez, de manera positiva, con la edad $(r=0.43)(P \leq$ $0.05)$, de manera que los árboles de mayor edad y altura, son los que muestran densidades más altas. En otras especies, como Pinus strobus, también se ha encontrado que la densidad de la madera depende principalmente de la edad (Hocker, 1984), lo cual es explicable, dado que a mayor edad se forman anillos con mayor porcentaje de madera tardía y ello incrementa la densidad de la madera en la parte más externa del árbol y por ende incrementa el valor promedio en los árboles más adultos (Daniel et al., 1982).

\section{CONCLUSIONES}

Los resultados del presente trabajo permiten concluir:

a) El valor promedio estimado de la densidad relativa (peso anhidro/ volumen verde) de la madera fue de $0.47 \mathrm{~g} / \mathrm{cm}^{3}$, lo que permite clasificarla como "moderadamente pesada".

b) De la variación total observada en la densidad de la madera, el $13 \%$ es atribuible a diferencias entre población, el $24 \%$ a diferencias entre árboles dentro de poblaciones y el $63 \%$ al error aleatorio, por lo que se puede seleccionar a nivel de población y entre árboles dentro de población. c) La densidad de la madera aumenta con la edad y altura del arbolado, dadas las correlaciones positivas encontradas entre estas variables dasométricas.

\section{RECONOCIMIENTOS}

Se agradece al Ing. Celestino Flores López por su colaboración en el trabajo de campo, al Dr. Jorge S. Marroquín de la Fuente por la revisión del manuscrito y al Dr. Eladio H. Cornejo Oviedo por la revisión y traducción del resumen.

\section{REFERENCIAS}

Becerra O., E.C y A. Plancarte B. 1993. Propagación asexual en Pinus greggii, injertos enraizados de estacas y acodos aéreos. Agrociencia. Recursos renovables 3(1):97-110.

Beristain B., J.J. 1992. Variación morfológica y anatómica de acículas de Pinus greggii Engelm. Tesis profesional. Universidad Autónoma Chapingo. Chapingo, Mex. 93 p.

Burley J. y P.J. Wood (comp). 1979. Manual sobre investigacioones de especies y procedencias con referencia especial a los trópicos. Department of Forestry. Commonwealth Forestry Institute. University of Oxford. Tropical Forestry Papers No.10. 233 p. + apéndices.

Cetina A., V.M., V.A. González. A. y J.J. Vargas H. 1999. El manejo en vivero de Pinus greggii Engelm. y la calidad de la planta. Agrociencia. 33(4):423430.

Capó A., M.A., R. López A. y E. Cornejo O. 1993. Crecimiento de Pinus greggii en suelo de ocho localidades. In: Resúmenes del I congreso 
mexicano sobre recursos naturales. Saltillo, Coah. 75 p.

Daniel T., W., J.A. Helms y F.S. Baker. 1982. Principios de silvicultura. Mc Graw-Hill. México. 492 p.

Donahue J., K. y J. López U. 1995. Geographic variation in leaf, cone and seed morphology of Pinus greggii in native forests. CAMCORE. Cooperative Department of Forestry, North Carolina State University. EUA. $10 \mathrm{p}$.

Donahue J., K. y J. López U. 1999. A new variety of Pinus greggii (PINACEAE) in México. SIDA Contribution to Botany. 18(4): 1083-1094.

Echenique M., R. y V. Díaz G. 1972. Algunas características tecnológicas de la madera de 11 especies mexicanas. Boletín Técnico. Núm. 27. INIF. México. 71 p.

Hernández M., E. 1995. Prueba de progenie de Pinus greggii Engelm., Procedencia Los Lirios, en el C.A.E.S.A., Arteaga, Coahuila. Tesis profesional. Universidad Autónoma Agraria Antonio Narro. Buenavista, Saltillo, Coah. 59 p.

Hocker Jr., H.W. 1984. Introducción a la biología forestal. A.G.T. Editor, S.A. México. $431 \mathrm{p}$.

Ibarra G., R. 1999. Variación de dimensiones transversales de traqueidas de madera tardía dentro y entre árboles de Pinus rudis Endl., en sierra las Alazanas, Arteaga, Coah. Tesis profesional. Universidad Autónoma Agraria Antonio Narro. Buenavista, Saltillo, Coah. 70 p.

INEGI. 1975. Carta topográfica. San José de Raíces G14C66. Escala 1: 50,000 .
INEGI. 1980. Carta topográfica. Ascensión G14C77. Escala $1: 50,000$.

INEGI. 1992a. Carta topográfica. Arteaga G14C34. Escala 1:50,000.

INEGI. 1992b. Carta topográfica. San Antonio de las Alazanas. Escala $1: 50,000$.

INEGI. 1993. Carta topográfica. Galeana G14C56. Escala 1:50,000.

Kollmann, F. 1959. Tecnología de la madera y sus aplicaciones. Instituto Forestal de Investigaciones y Experiencias y Servicio de la Madera. Madrid, España. 675 p.

Kietzka E.J., N.P. Denison. y W.S. Dvorak. 1996. Pinus greggii. New species for South Africa. Tree Improvement for Sustainable Tropical Forestry: 42-45.

López A., F. 1997. Variación en la densidad de la madera entre y dentro de árboles en Pinus rudis Endl., en sierra las Alazanas, Arteaga, Coah. Tesis profesional. Universidad Autónoma Agraria Antonio Narro. Saltillo, Coah. 55 p.

López U., J. y J.K. Donahue. 1995. Seed production of Pinus greggii Engelm. in natural Stands in México. CAMCORE. Cooperative, North Carolina. Tree Planter's. NC State University. North Carolina, EUA. 8692.

Malan S., F. 1994. The wood properties and quality of Pinus pringlei Shaw and $P$. greggii Engelm. Compared with that of $P$. patula and $P$. elliottii grown in South Africa. Division of Forest Science and Technology. SCIRD. 43-52.

Martínez, M. 1948. Los Pinos mexicanos. Segunda Edición. Ediciones Botas. 
México. 431 p.

Nienstaedt, H. 1990. Importancia de la variación natural. in: Memoria sobre mejoramiento genético y plantaciones forestales. Centro de Genética Forestal, A.C. Chapingo, México. 16-23.

Ornelas H., G. 1997. Ensayo de tres procedencias de Pinus greggii Engelm., en el C.A.E.S.A., Arteaga, Coahuila. Tesis profesional. Universidad Autónoma Agraria Antonio Narro. Saltillo, Coah. 59 p.

Ordoñez C., V. G. Bárcenas P. y A. Quiroz S. 1989. Características físico-mecánicas de la madera de diez especies de San Pablo Macuiltianguis, Oaxaca. Boletín Técnico. La madera y su uso. No. 21. Instituto de Ecología, A.C. Xalapa, Veracruz. 30 p.

Perry Jr., J.P. 1991. The pines of México and Central America. Timber Press, Inc. Portland, Oregon, EUA. 563 p.

Poynton, J. R. 1977. Tree planting in Southern Africa, the pines. Vol. 1. Department of Forestry. South Africa. 576 p.

Plancarte B., A. 1990. Variación en longitud de conos y peso de semillas en Pinus greggii Engelm. de tres procedencias de Hidalgo y Querétaro. Centro de Genética Forestal, A.C. Nota técnica No. 4. Chapingo, Edo. de México: 1-6.

Ramírez H., C. 1993. Evaluación de la diversidad genética en poblaciones naturales de Pinus greggii Engelm. Tesis de Maestría en Ciencias. Colegio de postgraduados, Montecillo, Edo. de México. 90 p.

Rodríguez V., E. 1998. Variación de largo de traqueidas dentro y entre árboles de Pinus rudis Endl., de sierra las
Alazanas, Mpio. de Arteaga, Coah. Tesis profesional. Universidad Autónoma Agraria Antonio Narro. Saltillo, Coah. 52 p.

Santos G., R. 1998. Variación morfológica y anatómica en hojas y conos de ocho localidades de Pseudotsuga del Norte de México. Tesis profesional. Universidad Autónoma Agraria Antonio Narro. Saltillo, Coah.

Serrato C., J.A. 2000. Prueba de progenie de Pinus greggii Engelm. en el predio los Tarihuanes, Cañón de Jamé, Arteaga, Coah. Tesis profesional. Universidad Autónoma Agraria Antonio Narro. Saltillo, Coah. $67 \mathrm{p}$.

Smith D. M. 1954. Maximun moisture content method por determining specific gravity of small wood samples. Forest Products Laboratory. Rept. No. 2014. USDA. Forest Service. Madison, Wis. EUA. $8 \mathrm{p}$.

SPP. 1981. Atlas Nacional del Medio físico.Carta climática, carta de temperatura, carta de precipitación. Escala 1:1000000. Secretaría de Programación y Presupuesto. México. 224 p.

Trujillo G., M.C. 1999. Variación de la densidad de la madera de Pinus teocote Schl. y Cham. en el ejido la Trinidad, Montemorelos, N.L. Tesis profesional. Universidad Autónoma Agraria Antonio Narro. Saltillo, Coah. $50 \mathrm{p}$.

Valencia M., S. y J.J. Vargas H. 1997. Método empírico para estimar la densidad básica en muestras pequeñas de madera. Madera y Bosques 3(1): 81-87.

Valencia M., S., J.J. Vargas H. y J.M. Chacón S. 1993. Un método sencillo para la estimación de la densidad de la madera. I Congreso Mexicano 
sobre los recursos forestales. Saltillo, Coah. México. 70p.

Valencia M.,S., J.J.Vargas H., J.D. Molina G. y J. Jasso M. 1996. Control genético de la velocidad de crecimiento y características de la madera en Pinus patula Schl. Agrociencia. 30: 265-273.

Vargas H., J.J. y A. Muñoz. O. 1988. Crecimiento y supervivencia en plantulas de cuatro especies de Pinus. Agrociencia 72:197-208.

Yáñez M., O. y M. Caballero D. 1991. Variación de algunas características de Pinus strobus var. chiapensis Mtz. de tres localidades de su distribución natural: densidad relativa y longitud de traqueidas de la madera. Revista Chapingo 15 (75):18-24.
Zobel, B. J. y T.J. Talbert. 1988. Técnicas de mejoramiento genético de árboles forestales. Editorial Limusa. México. $545 \mathrm{p}$.

Zobel, B.J. y J.P. Van Buijtenen. 1989. Wood variation, its causes and control. Springer Verlag. Alemania. $363 \mathrm{p}$.

Zobel, B. J., G. van Wik y P. Stahl. 1987. Growing Exotic Forest. John Wiley and Sons Ed. Nueva York, EUA. 508 p.

Zuñiga B., M.C. y S. Valencia M. 1999. Variación de la densidad de la madera de Pseudotsuga entre árboles y entre localidades del Norte de México. In: Resúmenes del IV Congreso Mexicano sobre Recursos Forestales. Durango, México. 119120.»

1 Departamento Forestal Universidad Autónoma Agraria Antonio Narro. Buenavista, Saltillo, Coah. México. 25315. c.e.: svalencia@narro.uaaan.mx.

Manuscrito recibido para su publicación el: 12 de julio de 2000

Aceptado el: 6 de octubre de 2000

Este documento se debe citar como:

López L., M. y S. Valencia M. Variación de la densidad relativa de la madera de Pinus greggii Engelm. del norte de México. Madera y Bosques 7(1):37-46. 
